\title{
Multivariate Analysis as a Tool for Studying the Effects of Salinity in Different Melon Landraces at Germination Stage
}

\author{
Behrooz SARABI ${ }^{1 *}$, Sahebali BOLANDNAZAR ${ }^{1}$, Nasser GHADERI ${ }^{2}$, \\ Seyed Jalal TABATABAEI ${ }^{3}$
}

\author{
IUniversity of Tabriz,Faculty of Agriculture,Department of Horticulture,Tabriz,Iran;sarabib@tabrizuacir("correspondingauthor); bolandnazar@tabrizu.aciv \\ ${ }^{2}$ University ofKurdistan, Faculty of Agriculture, Department of Horticultural Sciences, Sanandaj; Iran; n.ghaderi@uok.acir \\ ${ }^{3}$ University ofShahed, Faculty of Agriculture, Tehran Qome high way, Post Cod, 3319118651, Tehram, Iran;j.tabatabaei@shahed.acir
}

\begin{abstract}
Although salt stress affects all growth stages of a plant, seed germination and seedling growth stages are known to be more sensitive for most plant species. The current research was carried out to find the most effective indicators when screening melons for salt tolerance. For this purpose, a factorial experiment as a completely randomized design was performed in order to investigate the effects of salinity $(0,20,40,60$ and $80 \mathrm{mM} \mathrm{NaCl})$ on seed germination parameters, hydrogen peroxide content, lipid peroxidation and some antioxidant enzymes (guaiacol peroxidase, ascorbate peroxidase and superoxide dismutase) in five Iranian melon landraces and one melon cultivar 'Galia' F1 at early seedling growth stage. The 'Galia' F1 was included for comparison as a salt-tolerant cultivar. The data collected from all salinity treatments across six landraces were used for analysis of variance and principal component analysis (PCA) using SPSS software as well as for obtaining Pearson's correlation coefficients $(\mathrm{r})$ among all physiological parameters. Obtained results indicated that averaged all landraces across all treatments, increased $\mathrm{NaCl}$ significantly affected all the parameters measured. In all landraces, salt tolerance index (STI) decreased as the salt concentrations increased. The principal component analysis revealed variations among the traits and determined four main factors that explained $97.53 \%$ of the total variance. Simple correlation coefficient analyses showed the existence of significant positive and negative correlations among characteristics. Our study suggests that the salt tolerance index and principal component analysis can be used as accurate and easy indicators when screening melons for salt tolerance.
\end{abstract}

Keywords: antioxidant enzymes, correlation, physiological parameters, principal component analysis, salt tolerance index

\section{Introduction}

Melons (Cucumis melo L.; $2 n=2 x=24$ ), are important horticultural crops in tropical and subtropical regions, which are also grown extensively in temperate climates. Worldwide, more than 25 million tons of melons were produced in 2010, where China, Turkey and Iran were the major producers (FAO, 2010). In Iran, more than $50 \%$ of total vegetable production is related to cucurbits. In 2010, Iranian farmers grew almost 75,000 ha of melon with a total production of 1.31 million tons (FAO, 2010).

In many areas of the world increasing salinity of cropland has become a major constraint to agricultural production (Munns, 2002). Although salt stress affects all growth stages of a plant, seed germination and seedling growth stages are known to be more sensitive in most plant species (Cuartero et al., 2006). Seed germination is a key ecological and agronomic trait, which plays a critical role affecting the subsequent growth and production of higher plants. Therefore, understanding the underlying mechanisms that regulate seed germination under salinity is of vital importance for agriculture. Recently, investigations have focused more on the mechanisms of salt tolerance in plants (Munns and Tester, 2008).

Seed germination in a salty medium is often used as an index of resistance to salinity stress in several crops. Difference in salt tolerance has been reported among muskmelon cultivars (Mangal et al., 1988; Mendlinger and Pasternak, 1992). Tests are needed each year to evaluate the salt tolerance of new and untested melon cultivars under various cultural conditions (Franco et al., 1997). Several authors reported that melons displays a moderate, though variable tolerance to salinity (Mangal et al., 1988; Meiri et al., 1982).

Reactive oxygen species (ROS) are formed in biological systems as part of normal metabolism. Under stress, enormous amounts of reactive oxygen species are produced that can cause peroxidation, resulting in damage to cell membranes, protein oxidation, enzyme inhibition and strand breakage in nucleic 
acids (Allen, 1995). Plants have developed elaborate mechanisms to detoxify these ROS and in these mechanisms, antioxidant enzymes play a very important role (Khanna-Chopra and Selote, 2007). Guaiacol peroxidase (POD), ascorbate peroxidase (APX) and superoxide dismutase (SOD) play a vital role in combating oxidative stress (Loggini et al., 1999).

In relation with Iranian melons, there are a few studies to assess potential variability for salinity tolerance over a wide spectrum of the gene pool at the seed germination stage and early seedling growth. This study aimed to determine the most tolerant and susceptible melon landraces to salinity with using the multivariate analysis in order to utilize them in the future melon breeding and genetic studies. For this purpose, we used multivariate analysis for defining the effective indicators when screening melons for salt tolerance and also to increase our understanding of the responses of Iranian landraces and their relationship with one resistance melons cultivar. To address this goal, five different Iranian melon landraces across the country, initially described by Pitrat et al. (2000), were selected. These landraces are under cultivation for hundreds of years in arid and semi arid regions of Iran, which salinity is the one factor limiting the growth and production of agricultural products. The 'Galia' F1 as a salt-tolerant cultivar (Yasar et al., 2006), was included for comparison. The effects of salinity stress on seed germination parameters, hydrogen peroxide content, lipid peroxidation and some antioxidant enzymes (POD, APX and SOD) in these landraces were studied.

\section{Materials and Methods}

\section{Plantmaterials}

Seeds of five different Iranian melon landraces from geographically diverse areas of the country were used. These landraces are under cultivation for hundreds of years as the most popular and commercial Iranian melons and have genetic stability in most traits. The 'Galia' F1 (New American Seed Company, USA) was included for comparison. Details about the origin and classification of landraces into groups are given in Table 1 .

\section{Seedgermination and sampling}

Seeds were disinfected in 1\% (active ingredient) sodium hypochlorite solution for $10 \mathrm{~min}$ to eliminate possible seedborne microorganisms, rinsed with sterile distilled water several times prior to drying for $30 \mathrm{~min}$ at room temperature (Korkmaz, 2005). Three replicates of 25 seeds were transferred into $10 \mathrm{~cm}$ sterile Petri dishes on filter paper and then were wetted with 10 $\mathrm{ml}$ distilled water (control) or saline water solution at 20, 40,60 and $80 \mathrm{mM} \mathrm{NaCl}$ with electrical conductivities (EC) of 1.95 , 3.69, 5.5 and $7.29 \mathrm{dS} \mathrm{m} \mathrm{m}^{-1}$, respectively. The Petri dishes were incubated in a growth chamber at $25 \pm 2^{\circ} \mathrm{C}$ and relative humidity of $65 \pm 5 \%$. Computation of germinated seeds was done daily until the numbers were stabilized. A seed scored germinated when radicle length reached $2 \mathrm{~mm}$.

At the end of experiment, the shoot and root length, shoot and root fresh weight, shoot and root dry weight, seedling fresh and dry weight, germination percent, germination rate, mean germination time (MGT), seed vigour indicator (SVI) and salt tolerance index (STI) were recorded. The remains of seedlings, including hypocotyl and cotyledons (plumule) were frozen in liquid nitrogen and stored under $-80{ }^{\circ} \mathrm{C}$ until biochemical analysis.

From the total number of seeds germinated, germination percentage (GP), Germination rate (GR) and mean germination time (MGT), an inverse measure of germination rate, were calculated according to the following formulas.

$$
\mathrm{GP}=\left(\frac{\text { Number of germinated seeds }}{\text { Total of number seeds }}\right) \times 100
$$

Germination rate $(\mathrm{GR})=\Sigma \frac{S_{i}}{D_{i}}$

Where: $S_{i}$ is the number of germinated seeds and $D_{i}$ is the day corresponding to $S_{i}$ (Bajji et al., 2002).

$$
\mathrm{MGT}=\frac{A_{1} D_{1}+A_{2} D_{2}+\ldots A_{n} D_{n}}{A_{1}+A_{2}+\ldots A_{n}}
$$

Where: $A$ is the number of seeds germinating per day; $D$ is the time corresponding to $A$ in days and $n$ is the number of days to final count (Cantliffe, 1991).

The seed vigour index was calculated according to the following equation:

$\mathrm{VI}=(\mathrm{SL}+\mathrm{RL}) \times \mathrm{GP}$

Where: SL is the shoot length; RL is the root length and GP is the germination percentage (Abdul-Baki and Anderson, 1973).

Salt tolerance index (STI) is the ratio of the seedling dry weight of the control treatment and seedling dry weight of the salt treatments. The STI was calculated from the following relation (El Goumi et al., 2014):

$$
\text { STI } \%=\left(\frac{\text { Total DW salt stress }}{\text { Total DW control }}\right) \times 100
$$

\section{Measurement of hydrogen peroxide $\left(\mathrm{H}_{2} \mathrm{O}_{2}\right)$ content}

The content of $\mathrm{H}_{2} \mathrm{O}_{2}$ was assayed using the method previously described by Alexieva et al., (2001). Approximately $0.25 \mathrm{~g}$ of frozen samples was extracted with $5 \mathrm{ml} 0.1 \%(\mathrm{w} / \mathrm{v})$ trichloroacetic acid (TCA) on ice. The homogenate was then transferred into a tube and centrifuged at $12,000 \mathrm{rpm}$ for $20 \mathrm{~min}$.

\begin{tabular}{|c|c|c|c|c|c|c|}
\hline Accessions no. & Plant designation & Collecting sites & Cultivar group & Latitude $\left({ }^{\circ}\right)$ & Longitude $\left({ }^{\circ}\right)$ & 1000 seed weight $(\mathrm{g})$ \\
\hline 1 & Samsuri & Varamin & cantalupensis & 35 & 51 & 50.87 \\
\hline 2 & Kashan & Kashan & cantalupensis & 33 & 51 & 65.33 \\
\hline 3 & Khatouni & Mashhad & inodorus & 36 & 59 & 62.64 \\
\hline 4 & Suski-e-Sabz & Eyvanki & inodorus & 35 & 52 & 62.01 \\
\hline 5 & Ghobadlu & Ajab Shir & inodorus & 37 & 45 & 42.54 \\
\hline 6 & ‘Galia' F1 Hybrid & - & reticulatus & - & - & 33.26 \\
\hline
\end{tabular}
In order to determine the concentration of $\mathrm{H}_{2} \mathrm{O}_{2}, 250 \mu \mathrm{l}$ of the

Table 1. Melon landraces used in this study

Plant designation indicates the common name and the cultivar group, according Pitrat et al. (2000). 
266

supernatant was added to $250 \mu \mathrm{l}$ potassium phosphate buffer $(100 \mathrm{mM}, \mathrm{pH} 7.0)$ and $500 \mu \mathrm{l} \mathrm{KI}(1 \mathrm{M})$. The absorbance of supernatant was read at $390 \mathrm{~nm}$. The content of $\mathrm{H}_{2} \mathrm{O}_{2}$ was assayed by a standard curve and expressed as $\mathrm{nMg}^{-1} \mathrm{DW}$.

\section{Lipidperoxidation}

Membrane lipid peroxidation was characterised by the main product of the lipid peroxidation, the malondialdehyde (MDA) concentration, measured by the thiobarbituric acid (TBA) assay (Heath and Packer, 1968). Samples of $0.25 \mathrm{~g}$ were homogenized with $1.5 \mathrm{ml}$ of $0.1 \%$ trichloroacetic acid (TCA) for $1 \mathrm{~min}$ on ice. The homogenate was centrifuged at 14,000 rpm for $15 \mathrm{~min}$ at 4 ${ }^{\circ}$ C. The supernatant $(0.5 \mathrm{ml})$ was mixed with $0.5 \mathrm{ml}$ of $0.5 \%$ TBA diluted with $20 \%$ of TCA. The mixed was incubated at 95 ${ }^{\circ} \mathrm{C}$ for $30 \mathrm{~min}$ and then placed on ice to stop the reaction. After that, the homogenate was again centrifuged at $10,000 \mathrm{rpm}$ for 10 $\min$ at $4{ }^{\circ} \mathrm{C}$. The absorbance of the supernatant at $532 \mathrm{~nm}$ was corrected for non-specific absorbance by subtracting the absorbance at $600 \mathrm{~nm}$. Concentration of MDA was estimated by the extinction coefficient $\left(155 \mathrm{mM}^{-1} \mathrm{~cm}^{-1}\right)$. Three replicates were measured per salinity treatment and landrace.

\section{Protein assay}

Total soluble protein was determined by dye binding technique (Bradford, 1976) using bovine serum albumin as protein standard.

\section{Guaiacolperoxidase (POD)}

The guaiacol peroxidase (POD; EC 1.11.1.7) activity was determined as described by Hemeda and Kelin (1990) in a reaction mixture $(1 \mathrm{ml})$ containing $50 \mathrm{mM}$ potassium phosphate buffer ( $\mathrm{pH} 6.6), 1 \%$ guaiacol, $0.3 \% \mathrm{H}_{2} \mathrm{O}_{2}$ and $20 \mu \mathrm{l}$ enzyme extract. The addition of enzyme extract started the reaction and the increase in absorbance was recorded at $470 \mathrm{~nm}$ for 2 min. Enzyme activity was quantified by the amount of tetraguaiacol formed using its molar extinction coefficient (26.6 $\left.\mathrm{mM}^{-1} \mathrm{~cm}^{-1}\right)$ and the enzyme activity was expressed as $\mu \mathrm{M} \mathrm{min}^{-1}$ $\mathrm{mg}^{-1}$ protein.

\section{Ascorbateperoxidase (APX)}

The activity of ascorbate peroxidase (APX; EC 1.11.1.11) was measured using the method of Nakano and Asada (1981). The reaction mixture consisted of $100 \mathrm{mM}$ potassium phosphate buffer $(\mathrm{pH} 7)$ containing $10 \mathrm{mM}$ ascorbic acid and crude enzyme extract. The reaction was started by the addition of $\mathrm{H}_{2} \mathrm{O}_{2}$ at a final concentration of $0.2 \mathrm{mM}$. Oxidation of ascorbic acid as a decrease in absorbance at $290 \mathrm{~nm}$ was followed $2 \mathrm{~min}$ after starting the reaction. The difference in absorbance was divided by the ascorbate molar extinction coefficient $\left(2.8 \mathrm{mM}^{-1}\right.$ $\left.\mathrm{cm}^{-1}\right)$ and the enzyme activity was expressed as $\mu \mathrm{M} \mathrm{min}{ }^{-1} \mathrm{mg}^{-1}$ protein.

\section{Superoxide dismutase (SOD)}

The superoxide dismutase (SOD; EC 1.15.1.1) activity was measured as described by Dhindsa et al. (1981). The reaction mixture contained $50 \mathrm{mM}$ potassium phosphate buffer $(\mathrm{pH}$ 7.8), $13 \mathrm{mM}$ methionine, $75 \mu \mathrm{M}$ nitroblue tetrazolium (NBT), $2 \mu \mathrm{M}$ riboflavin, $0.1 \mathrm{mM}$ EDTA and the $20 \mu \mathrm{l}$ of leaf extract. The A560 was recorded after a 15 min illumination period. In this assay, 1 unit of SOD is defined as the amount required to inhibit the photoreduction of NBT by $50 \%$.

\section{Statistical analysis}

A factorial experiment was performed as a completely randomized design with three replications $(25$ seeds per Petri dish). The first factor was six landraces of melon (Khatouni, Suski-Sabz, Kashan, Samsuri, Ghobadlu and 'Galia' F1) and five levels of salinity $(0,20,40,60$ and $80 \mathrm{mM} \mathrm{NaCl})$. The data obtained were subjected to statistical analysis using SPSS 16.0 software (SPSS Inc., IBM) and when significant differences were observed, Duncan's Multiple Range Test was used to determine significance of differences between variables $(p<0.05)$. The data collected from all salinity treatments across six landraces were used for principal component analysis (PCA) using SPSS software as well as for obtaining Pearson's correlation coefficients (r) among all physiological parameters.

\section{Results}

\section{Salinity effects on germination traits and early seedling growth}

In general, salinity affected all the parameters measured. Increasing $\mathrm{NaCl}$ significantly decreased the shoot and root length, shoot and root fresh weight, shoot and root dry weight, seedling fresh and dry weight, germination percent, germination rate and seed vigour indicator but increased mean germination time (MGT) in all landraces (Table 2).

Regarding table 2, shoot fresh weight, seedling fresh weight and mean germination time (MGT) were not significantly affected by $\mathrm{NaCl} \leq 20 \mathrm{mM}$, however at $40 \mathrm{mM}$ to $80 \mathrm{mM}$ salinity, shoot and seedling fresh weight were progressively reduced, while MGT was significantly increased. Significant

Table 2. Effects of salinity treatments on germination traits across all melon landraces

\begin{tabular}{|c|c|c|c|c|c|c|c|c|c|c|c|c|}
\hline Treatments & $\begin{array}{l}\text { Shoot } \\
\text { length } \\
(\mathrm{mm})\end{array}$ & $\begin{array}{l}\text { Root } \\
\text { length } \\
(\mathrm{mm})\end{array}$ & $\begin{array}{l}\text { Shoot } \\
\text { fresh } \\
\text { weight } \\
(\mathrm{g})\end{array}$ & $\begin{array}{l}\text { Root } \\
\text { fresh } \\
\text { weight } \\
\text { (g) }\end{array}$ & $\begin{array}{c}\text { Shoot } \\
\text { dry } \\
\text { weight } \\
\text { (g) }\end{array}$ & $\begin{array}{c}\text { Root } \\
\text { dry } \\
\text { weight } \\
(\mathrm{g})\end{array}$ & $\begin{array}{l}\text { Seedling } \\
\text { fresh } \\
\text { weight } \\
\text { (g) }\end{array}$ & $\begin{array}{l}\text { Seedling } \\
\text { dry } \\
\text { weight } \\
\text { (g) }\end{array}$ & $\begin{array}{l}\text { Germination } \\
\text { percent (\%) }\end{array}$ & $\begin{array}{c}\text { Germination } \\
\text { rate } \\
(\text { Germinated } \\
\left.\text { seeds day }{ }^{-1}\right) \\
\end{array}$ & $\begin{array}{l}\text { Seed vigour } \\
\text { indicator }\end{array}$ & $\begin{array}{c}\text { Mean } \\
\text { germination } \\
\text { time (day) }\end{array}$ \\
\hline Control & $73.25^{\mathrm{a}}$ & $70.24^{\mathrm{a}}$ & $0.26^{\mathrm{a}}$ & $0.074^{a}$ & $0.029^{\mathrm{a}}$ & $0.0063^{\mathrm{a}}$ & $0.33^{\mathrm{a}}$ & $0.035^{\mathrm{a}}$ & $95.07^{\mathrm{a}}$ & $5.22^{\mathrm{a}}$ & $13625.55^{a}$ & $2.84^{\mathrm{c}}$ \\
\hline $20 \mathrm{mM} \mathrm{NaCl}$ & $67.64^{\mathrm{ab}}$ & $69.40^{\mathrm{a}}$ & $0.24^{\mathrm{a}}$ & $0.067^{\mathrm{ab}}$ & $0.026^{\mathrm{b}}$ & $0.0059^{\mathrm{ab}}$ & $0.31^{\mathrm{a}}$ & $0.032^{\mathrm{b}}$ & $93.5^{\mathrm{ab}}$ & $5.18^{\mathrm{a}}$ & $12835.86^{\mathrm{ab}}$ & $2.84^{c}$ \\
\hline $40 \mathrm{mM} \mathrm{NaCl}$ & $64.94^{\mathrm{b}}$ & $64.98^{\mathrm{ab}}$ & $0.22^{\mathrm{b}}$ & $0.058^{\mathrm{bc}}$ & $0.026^{\mathrm{b}}$ & $0.0050^{\mathrm{bc}}$ & $0.28^{\mathrm{b}}$ & $0.031^{\mathrm{b}}$ & $91.53^{\mathrm{ab}}$ & $4.90^{\mathrm{ab}}$ & $11914.07^{\mathrm{bc}}$ & $3.05^{\mathrm{b}}$ \\
\hline $60 \mathrm{mM} \mathrm{NaCl}$ & $62.20^{\mathrm{b}}$ & $57.57^{b}$ & $0.22^{\mathrm{bc}}$ & $0.055^{c}$ & $0.023^{c}$ & $0.0040^{\mathrm{d}}$ & $0.27^{\mathrm{bc}}$ & $0.028^{c}$ & $90.26^{b}$ & $4.72^{\mathrm{b}}$ & $10819.52^{\mathrm{cd}}$ & $3.21^{\mathrm{ab}}$ \\
\hline $80 \mathrm{mM} \mathrm{NaCl}$ & $60.27^{b}$ & $56.53^{b}$ & $0.20^{c}$ & $0.054^{c}$ & $0.023^{c}$ & $0.0043^{\text {cd }}$ & $0.25^{\mathrm{c}}$ & $0.027^{c}$ & $89.87^{b}$ & $4.65^{\mathrm{b}}$ & $10557.55^{d}$ & $3.29^{\mathrm{a}}$ \\
\hline \multicolumn{13}{|l|}{ Significance } \\
\hline Landraces (L) & $* *$ & $*$ & $* * *$ & $* *$ & $* * *$ & $* * *$ & $* * *$ & $* * *$ & $*$ & $* *$ & $* * *$ & $* * *$ \\
\hline $\begin{array}{c}\text { Salinity } \\
\text { treatments }(S)\end{array}$ & $* * *$ & $* * *$ & $* * *$ & $* * *$ & $* * *$ & $* * *$ & $* * *$ & $* * *$ & $* * *$ & $* * *$ & $* * *$ & $* * *$ \\
\hline $\mathrm{L} \times \mathrm{S}$ & NS & NS & NS & NS & NS & NS & NS & NS & $*$ & $* * *$ & NS & NS \\
\hline
\end{tabular}

Values with different superscripts within the same column show significant differences at $P<0.05$ level between treatments (Duncan's multiple range test).

NS, $\left({ }^{*}\right),\left({ }^{* *}\right),\left({ }^{* *}\right)$ are non-significant or significant at $P<0.05,0.01$ or 0.001 , respectively. 
decreases in shoot length, root fresh and dry weight and seed vigour indicator (SVI) were found starting at $40 \mathrm{mM} \mathrm{NaCl}$, compared to the non-salinity control. So that, seed vigour indicator significantly decreased $12.56 \%, 20.59 \%$ and $22.52 \%$ at 40,60 and $80 \mathrm{mM} \mathrm{NaCl}$, respectively, compared to the nonsalinity control.

The highest SVI was observed in the Samsuri landrace, that had the highest shoot and root length among all investigated landraces. Also, decreases in root length, germination percent and germination rate were found starting at $60 \mathrm{mM} \mathrm{NaCl}$, significantly, compared to the control. For example, root length significantly decreased $18.03 \%$ and $19.76 \%$ at 60 and $80 \mathrm{mM}$ $\mathrm{NaCl}$, respectively, compared to the non-salinity treatment.

According to the salt tolerance index (STI), the Ghobadlu landrace had the highest index (97.57\%) that was followed by Suski-e-Sabz, Kashan, Khatouni, Samsuri and 'Galia' F1, 89.83\%, $89.13 \%, 83.68 \%, 78.7 \%$ and $68.64 \%$, respectively (Fig. 1 ).

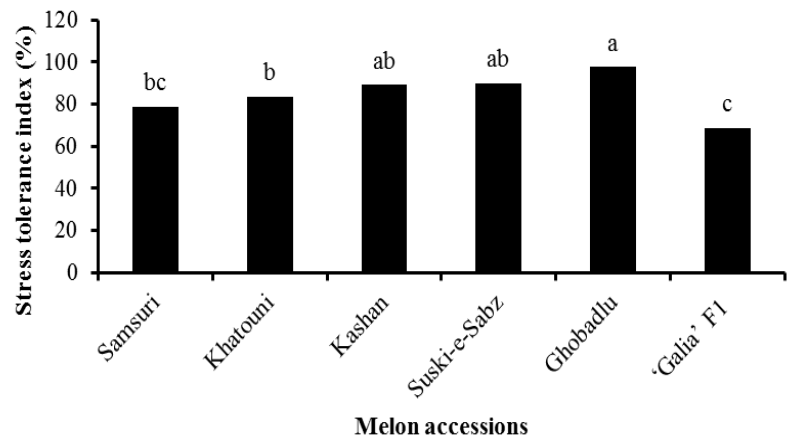

Fig. 1. Effects of salinity treatments on salt tolerance index in germinated seeds of melon landraces. The different letters represent significant differences at $5 \%$ probability, by Duncan's Multiple Range test
Salinity effects on hydrogen peroxide content, lipid peroxidation and activities of antioxidant enzymes

Averaged across all landraces, increased $\mathrm{NaCl}$ generally increased $\mathrm{H}_{2} \mathrm{O}_{2}$ content, lipid peroxidation and activities of antioxidant enzymes (Table 3). Hydrogen peroxide, lipid peroxidation, POD, APX and SOD activities increased 70.45\%, $8.39,2.11,2.13$ and 2.11 fold for $80 \mathrm{mM} \mathrm{NaCl}$, respectively; compared to the control (Table 3).

Across all salinity treatments, the landraces differed significantly in content of $\mathrm{H}_{2} \mathrm{O}_{2}$, lipid peroxidation and activities of antioxidant enzymes (Table 4). Among all landraces across all

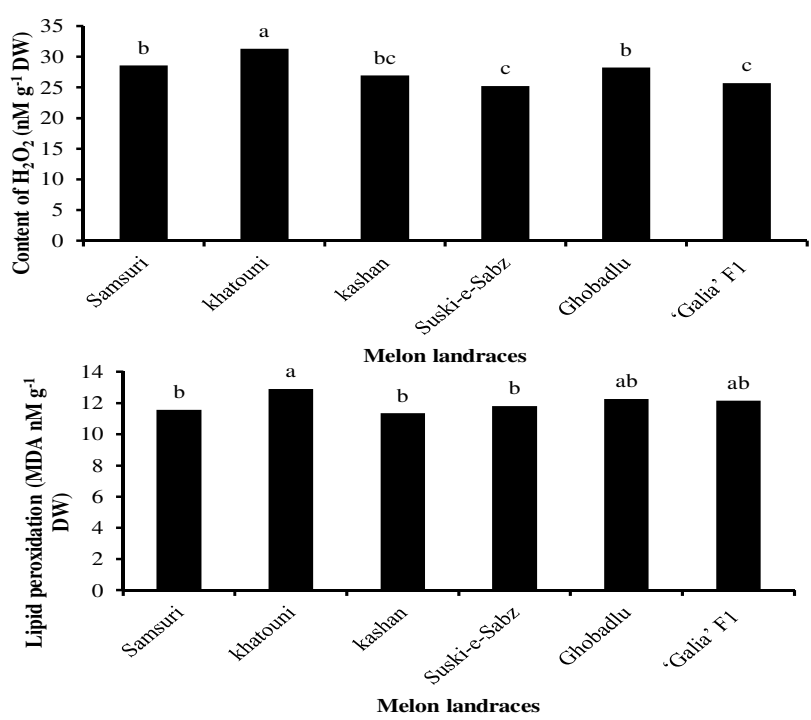

Fig. 2. Effects of salinity treatments on hydrogen peroxide $\left(\mathrm{H}_{2} \mathrm{O}_{2}\right)$ content and lipid peroxidation in germinated seeds of melon landraces. The same letters did not differ significantly at $5 \%$ probability, by Duncan's Multiple Range test

Table 3. Effects of salt stress on hydrogen dioxide $\left(\mathrm{H}_{2} \mathrm{O}_{2}\right)$ content, lipid peroxidation, antioxidant enzymes activities including guaiacol peroxidase (POD), ascorbate peroxidase (APX) and superoxide dismutase (SOD) across all melon landraces

\begin{tabular}{|c|c|c|c|c|c|}
\hline Treatments & $\begin{array}{l}\text { Content of } \mathrm{H}_{2} \mathrm{O}_{2} \\
\left(\mathrm{nM} \mathrm{g}^{-1} \mathrm{DW}\right)\end{array}$ & $\begin{array}{l}\text { Lipid peroxidation } \\
\left(\mathrm{MDA} \mathrm{nM} \mathrm{g}^{-1} \mathrm{DW}\right)\end{array}$ & $\begin{array}{c}\text { POD activity }(\mu \mathrm{M} \text { min } \\
\left.{ }^{1}{ }^{-1}{ }^{-1} \text { protein }\right)\end{array}$ & $\begin{array}{l}\text { APX activity }(\mu \mathrm{M} \\
\left.\min ^{-1} \mathrm{mg}^{-1} \text { protein }\right)\end{array}$ & $\begin{array}{c}\text { SOD activity }\left(\mathrm{U} \mathrm{mg}^{-1}\right. \\
\text { protein) }\end{array}$ \\
\hline Control & $20.68^{e}$ & $2.71^{\mathrm{e}}$ & $116.97^{\mathrm{c}}$ & $63.22^{\mathrm{d}}$ & $0.774^{\mathrm{e}}$ \\
\hline $20 \mathrm{mM} \mathrm{NaCl}$ & $24.62^{d}$ & $5.84^{\mathrm{d}}$ & $150.15^{b c}$ & $73.36^{\mathrm{cd}}$ & $0.927^{\mathrm{d}}$ \\
\hline $40 \mathrm{mM} \mathrm{NaCl}$ & $26.42^{c}$ & $10.87^{\mathrm{c}}$ & $150.62^{b c}$ & $79.21^{\mathrm{c}}$ & $1.207^{\mathrm{c}}$ \\
\hline $60 \mathrm{mM} \mathrm{NaCl}$ & $31.41^{\mathrm{b}}$ & $17.87^{\mathrm{b}}$ & $183.52^{b}$ & $111.21^{\mathrm{b}}$ & $1.446^{\mathrm{b}}$ \\
\hline $80 \mathrm{mM} \mathrm{NaCl}$ & $35.25^{\mathrm{a}}$ & $22.73^{\mathrm{a}}$ & $247.18^{\mathrm{a}}$ & $134.75^{a}$ & $1.630^{\mathrm{a}}$ \\
\hline \multicolumn{6}{|l|}{ Significance } \\
\hline Landraces $(\mathrm{L})$ & $* * *$ & $*$ & $* * *$ & $* * *$ & $* * *$ \\
\hline Salinity treatments (S) & $* * *$ & $* * *$ & $* * *$ & $*$ & $* * *$ \\
\hline $\mathrm{L} \times \mathrm{S}$ & $* * *$ & $* * *$ & $* * *$ & $* * *$ & $* * *$ \\
\hline
\end{tabular}

Means followed by the same letter within a column for a given trait were not significantly different at $P<0.05$.

$\left({ }^{*}\right),\left({ }^{* *}\right)$ are significant at $P<0.05$ or 0.001 , respectively.

Table 4. Landrace differences in hydrogen dioxide $\left(\mathrm{H}_{2} \mathrm{O}_{2}\right)$ content, lipid peroxidation, antioxidant enzymes activities including guaiacol peroxidase (POD), ascorbate peroxidase (APX) and superoxide dismutase (SOD) across all $\mathrm{NaCl}$ treatments

\begin{tabular}{|c|c|c|c|c|c|}
\hline Landraces & $\begin{array}{l}\text { Content of } \mathrm{H}_{2} \mathrm{O}_{2} \\
\left(\mathrm{nM} \mathrm{g}^{-1} \mathrm{DW}\right)\end{array}$ & $\begin{array}{l}\text { Lipid peroxidation } \\
\left(\mathrm{MDA} \mathrm{nM} \mathrm{g}^{-1} \mathrm{DW}\right)\end{array}$ & $\begin{array}{c}\text { POD activity }\left(\mu \mathrm{M} \min ^{-1}\right. \\
\left.\mathrm{mg}^{-1} \text { protein }\right)\end{array}$ & $\begin{array}{c}\text { APX activity }(\mu \mathrm{M} \text { min } \\
\left.{ }^{1} \mathrm{mg}^{-1} \text { protein }\right)\end{array}$ & $\begin{array}{c}\text { SOD activity (U mg }{ }^{-1} \\
\text { protein) }\end{array}$ \\
\hline Samsuri & $28.59^{\mathrm{b}}$ & $11.57^{\mathrm{b}}$ & $126.43^{\mathrm{c}}$ & $79.18^{\mathrm{b}}$ & $1.01^{\mathrm{e}}$ \\
\hline Khatouni & $31.34^{\mathrm{a}}$ & $12.90^{\mathrm{a}}$ & $173.27^{\mathrm{b}}$ & $103.07^{\mathrm{a}}$ & $1.21^{\mathrm{c}}$ \\
\hline Kashan & $26.92^{\mathrm{bc}}$ & $11.34^{\mathrm{b}}$ & $159.70^{\mathrm{bc}}$ & $102.32^{\mathrm{a}}$ & $1.30^{\mathrm{b}}$ \\
\hline Suski-e-Sabz & $25.24^{\mathrm{c}}$ & $11.80^{\mathrm{b}}$ & $138.91^{\mathrm{bc}}$ & $90.85^{\mathrm{ab}}$ & $1.12^{\mathrm{d}}$ \\
\hline Ghobadlu & $28.26^{\mathrm{b}}$ & $12.26^{\mathrm{ab}}$ & $334.91^{\mathrm{a}}$ & $95.77^{\mathrm{ab}}$ & $1.43^{\mathrm{a}}$ \\
\hline ‘Galia' F1 Hybrid & $25.70^{c}$ & $12.16^{\mathrm{ab}}$ & $84.94^{\mathrm{d}}$ & $82.94^{b}$ & $1.11^{\mathrm{d}}$ \\
\hline
\end{tabular}

Means followed by the same letter within a column for a given trait were not significantly different at $P<0.05$. 
268
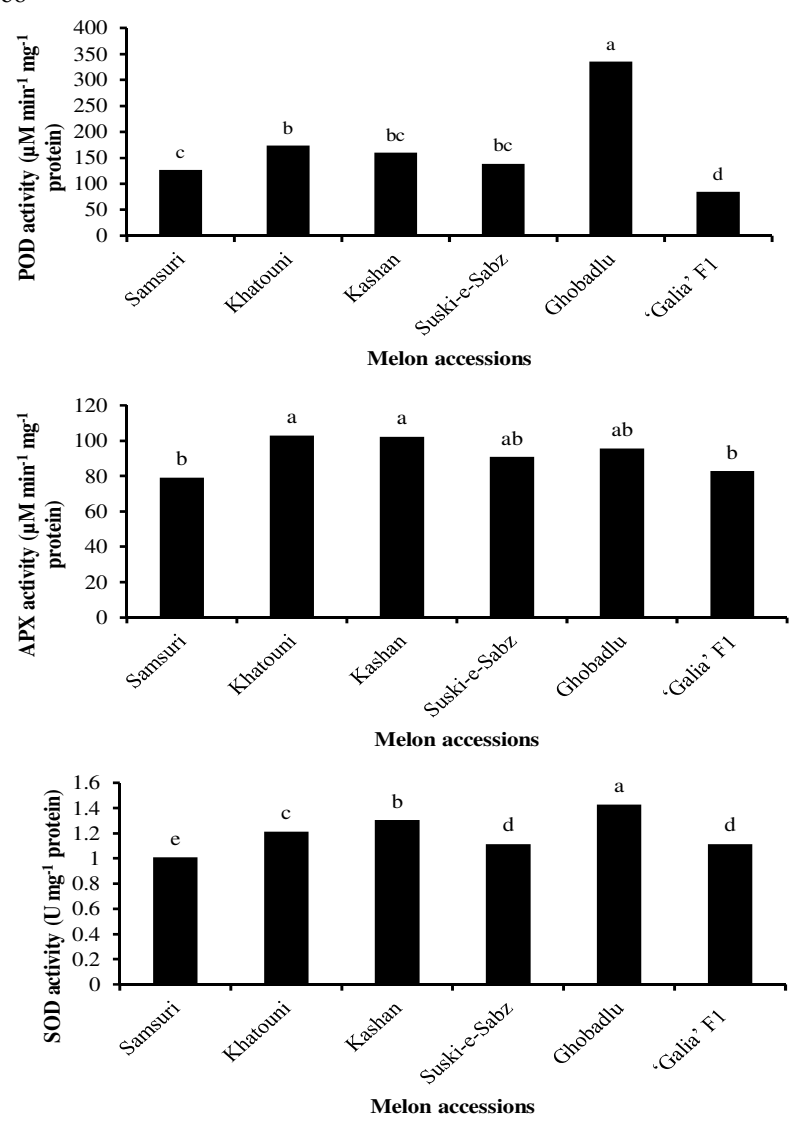

Fig. 3. Effects of salinity treatments on guaiacol peroxidase (A), ascorbate peroxidase (B) and superoxide dismutase (C) activities in germinated seeds of melon landraces. The different letters represent significant differences at 5\% probability, by Duncan's Multiple Range test

treatments, content of $\mathrm{H}_{2} \mathrm{O}_{2}$, lipid peroxidation, POD, APX and SOD activities ranged from 25.24 to $31.34 \mathrm{nM} \mathrm{g}^{-1} \mathrm{DW}$, 11.34 to $12.9 \mathrm{MDA} \mathrm{nM} \mathrm{g}^{-1} \mathrm{DW}, 84.94$ to $334.91 \mu \mathrm{M} \mathrm{min}^{-1} \mathrm{mg}^{-1}$ protein, 79.18 to $103.07 \mu \mathrm{M} \mathrm{min}^{-1} \mathrm{mg}^{-1}$ protein and 1.01 to 1.43 $\mathrm{Umg}^{-1}$ protein, respectively (Table 4).

The lowest $\mathrm{H}_{2} \mathrm{O}_{2}$ content and lipid peroxidation were observed in the Suski-e-Sabz and Kashan landraces, respectively
(Fig. 2). The highest POD activity was noticeably exhibited in Ghobadlu landrace (Fig. 3A) to the extent that it was $48.26 \%$, $52.32 \%, 58.52 \%, 62.25 \%$ and $74.64 \%$ more than Khatouni, Kashan, Suski-e-Sabz, Samsuri and 'Galia' F1 landraces, respectively.

\section{Correlations among traits}

Simple correlation coefficient analyses showed the existence of significant positive and negative correlations among characteristics (Table 5). For example, shoot dry weight and seedling dry weight, shoot fresh weight and seedling fresh weight, SOD and MDA, shoot dry weight and 1000 seed weight, seedling dry weight and 1000 seed weight, root length and seed vigour indicator had the highest positive correlations $(\mathrm{r}=$ $0.977^{* *}, 0.961^{* *}, 0.887^{* *}, 0.882^{* *}, 0.857^{* *}$ and $0.838^{* *}$, respectively). The negative correlations were also noted between mean germination time with germination rate and 1000 seed weight $\left(r=-0.896^{* *}\right.$ and $-0.712^{* *}$, respectively).

\section{PCA analysisfor traits}

The PCA analysis revealed variations among the traits and determined four main factors that explained $97.53 \%$ of the total variance (Table 6). For each factor, a loading value above 0.5 was considered as significant. The first principal component (PC1), second principal component (PC2), third principal component (PC3) and fourth principal component (PC4) accounted for $46.51 \%, 29.37 \%, 15.93 \%$ and 5.72 of observed variations, respectively.

The first principal component (PC1) and second principal component (PC2) that explained $75.88 \%$ of the total variance were applied to make a score plot (Fig. 4). According to score plot and ANOVA results at a significant level of $P<0.05$, the means of germination percent (99.11\%), POD activity (334.91 $\mu \mathrm{M} \mathrm{min}{ }^{-1} \mathrm{mg}^{-1}$ protein) and SOD activity (1.43 $\mathrm{U} \mathrm{mg}^{-1}$ protein) in the Ghobadlu landrace, that cultivated at the northwestern of Iran and eastern margin of Lake Urmia, were significantly higher than other landraces. The Khatouni landrace, a major Iranian winter melon that cultivated in the east of the country, had the highest APX activity (103.07 $\mu \mathrm{M} \mathrm{min}{ }^{-1} \mathrm{mg}^{-1}$ protein), $\mathrm{H}_{2} \mathrm{O}_{2}$ content $\left(31.34 \mathrm{nM} \mathrm{g}^{-1} \mathrm{DW}\right)$ and lipid peroxidation (12.9 MDA $\mathrm{nM} \mathrm{g}^{-1} \mathrm{DW}$ ) among all the landraces. In comparing with other

Table 5. Correlations among studied traits in melon landraces

\begin{tabular}{|c|c|c|c|c|c|c|c|c|c|c|c|c|c|c|c|c|c|c|c|}
\hline & SL & $\mathrm{RL}$ & SFW & RFW & SeFW & SDW & RDW & SeDW & GP & MGT & GR & SVI & $\mathrm{H}_{2} \mathrm{O}_{2}$ & SP & POD & APX & SOD & MDA & TSW \\
\hline SL & 1 & & & & & & & & & & & & & & & & & & \\
\hline $\mathrm{RL}$ & $0.347^{* *}$ & 1 & & & & & & & & & & & & & & & & & \\
\hline SFW & $0.508^{* *}$ & $0.440^{* *}$ & 1 & & & & & & & & & & & & & & & & \\
\hline RFW & $0.395^{* *}$ & $0.389^{* *}$ & $0.515^{* *}$ & 1 & & & & & & & & & & & & & & & \\
\hline SeFW & $0.532^{* *}$ & $0.476^{* *}$ & $0.961^{* *}$ & $0.733^{* *}$ & 1 & & & & & & & & & & & & & & \\
\hline SDW & $0.456^{* *}$ & $0.221^{*}$ & $0.672^{* *}$ & $0.218^{*}$ & $0.604^{* *}$ & 1 & & & & & & & & & & & & & \\
\hline RDW & $0.347^{* *}$ & $0.437^{* *}$ & $0.383^{* *}$ & $0.578^{* *}$ & $0.491^{* *}$ & $0.171^{\mathrm{ns}}$ & 1 & & & & & & & & & & & & \\
\hline SeDW & $0.506^{* *}$ & $0.302^{* *}$ & $0.716^{* *}$ & $0.334^{* *}$ & $0.677^{* *}$ & $0.977^{* *}$ & $0.370^{* *}$ & 1 & & & & & & & & & & & \\
\hline GP & $0.151^{\mathrm{ns}}$ & $0.158^{\mathrm{ns}}$ & $0.297^{* *}$ & $0.423^{* *}$ & $0.372^{* *}$ & $0.104^{\mathrm{ns}}$ & $0.257^{*}$ & $0.158^{\mathrm{ns}}$ & 1 & & & & & & & & & & \\
\hline MGT & $-0.286^{* *}$ & $-0.286^{* *}$ & $-0.634^{* *}$ & $-0.457^{* *}$ & $-0.651^{* *}$ & $-0.579^{* *}$ & $-0.175^{n s}$ & $-0.585^{* *}$ & $-0.510^{* *}$ & 1 & & & & & & & & & \\
\hline GR & $0.181^{\mathrm{m}}$ & $0.2^{\text {ns }}$ & $0.511^{* *}$ & $0.451^{* *}$ & $0.551^{* *}$ & $0.423^{* *}$ & $0.183^{\text {ז }}$ & $0.444^{* *}$ & $0.586^{* *}$ & $-0.896^{* *}$ & 1 & & & & & & & & \\
\hline SVI & $0.704^{* *}$ & $0.838^{* *}$ & $0.580^{* *}$ & $0.548^{* *}$ & $0.638^{* *}$ & $0.362^{* *}$ & $0.515^{* *}$ & $0.454^{* *}$ & $0.488^{* *}$ & $-0.446^{* *}$ & $0.380^{* *}$ & 1 & & & & & & & \\
\hline $\mathrm{H}_{2} \mathrm{O}_{2}$ & $-0.558^{* *}$ & $-0.222^{*}$ & $-0.516^{* *}$ & $-0.079^{1 x}$ & $-0.435^{* *}$ & $-0.636^{* *}$ & $-0.166^{16}$ & $-0.629^{* *}$ & $0.146^{\mathrm{rs}}$ & $0.125^{\mathrm{Is}}$ & $0.063^{1 \times}$ & $-0.339^{* *}$ & 1 & & & & & & \\
\hline $\mathrm{SP}$ & $0.461^{* *}$ & $0.345^{* *}$ & $0.458^{* *}$ & $0.280^{* *}$ & $0.455^{* *}$ & $0.369^{* *}$ & $0.370^{* *}$ & $0.418^{* *}$ & $0.172^{\mathrm{rs}}$ & $-0.271^{* *}$ & $0.126^{\mathrm{Is}}$ & $0.475^{* *}$ & $-0.557^{* *}$ & 1 & & & & & \\
\hline POD & $-0.354^{* *}$ & $-0.09^{\mathrm{s}}$ & $-0.145^{\mathrm{ns}}$ & $-0.003^{\text {ns }}$ & $-0.116^{\mathrm{rs}}$ & $-0.244^{*}$ & $-0.278^{* *}$ & $-0.290^{* *}$ & $0.154^{\mathrm{s}}$ & $-0.290^{* *}$ & $0.327^{* *}$ & $-0.18^{\mathrm{ns}}$ & $0.482^{* *}$ & $-0.417^{* *}$ & 1 & & & & \\
\hline APX & $-0.226^{*}$ & $-0.281^{* *}$ & $-0.185^{\mathrm{ns}}$ & $-0.2^{18}$ & $-0.213^{*}$ & $0.009^{\mathrm{ss}}$ & $-0.267^{*}$ & $-0.039^{1 x}$ & $-0.008^{\mathrm{m}}$ & $0.01^{\mathrm{ns}}$ & $-0.015^{\mathrm{ns}}$ & $-0.293^{* *}$ & $0.251^{*}$ & $-0.560^{* *}$ & $0.279^{* *}$ & 1 & & & \\
\hline SOD & $-0.450^{* *}$ & $-0.334^{* *}$ & $-0.351^{* *}$ & $-0.217^{*}$ & $-0.350^{* *}$ & $-0.258^{*}$ & $-0.437^{* *}$ & $-0.331^{* *}$ & $-0.13^{\mathrm{m}}$ & $0.053^{\text {rs }}$ & $-0.009^{18}$ & $-0.461^{* *}$ & $0.549^{* *}$ & $-0.776^{* *}$ & $0.561^{* *}$ & $0.685^{* *}$ & 1 & & \\
\hline $\mathrm{MDA}$ & $-0.426^{* *}$ & $-0.302^{* *}$ & $-0.446^{* *}$ & $-0.276^{* *}$ & $-0.444^{* *}$ & $-0.336^{* *}$ & $-0.416^{* *}$ & $-0.399^{* *}$ & $-0.228^{*}$ & $0.262^{*}$ & $-0.172^{1 \mathrm{rs}}$ & $-0.454^{* *}$ & $0.538^{* *}$ & $-0.877^{* *}$ & $0.359^{* *}$ & $0.604^{* *}$ & $0.887^{* *}$ & 1 & \\
\hline TSW & $0.436^{* *}$ & $0.226^{*}$ & $0.726^{* *}$ & $0.269^{*}$ & $0.662^{* *}$ & $0.882^{* *}$ & $0.102^{\mathrm{rs}}$ & $0.857^{* *}$ & $0.163^{\mathrm{m}}$ & $-0.712^{* *}$ & $0.555^{* *}$ & $0.369^{* *}$ & $-0.517^{* *}$ & $0.193^{1 \mathrm{rs}}$ & $-0.04^{\mathrm{ns}}$ & $0.14^{\mathrm{s}}$ & $0.013^{\mathrm{n}}$ & $-0.135^{\mathrm{ns}}$ & 1 \\
\hline $\begin{array}{l}\text { Consid } \\
\text { (SDW) } \\
\text { indicate } \\
\text { Malono } \\
\left.\mathrm{NS},{ }^{*}\right)\end{array}$ & $\begin{array}{l}\text { (SVI), } \\
\text { aldehyd } \\
*^{* *} \text { ) are n }\end{array}$ & les: Sho & t length & $(\mathrm{SL}), \mathrm{R}$ & ot lengt & (RL), & $\begin{array}{l}\text { noot fres } \\
V) \text {, Gerr }\end{array}$ & h weigh & (SFW) & Root fr & resh wei & 18 & $\mathrm{FW}), \mathrm{Se}$ & eedling & $\begin{array}{l}\text { fresh w } \\
\text { ), Gerr } \\
\text { (APX), }\end{array}$ & ight (S & $\begin{array}{l}\text { FW), } \\
\text { rate ( } \\
\text { zide dis }\end{array}$ & $\begin{array}{l}\text { oot dry } \\
\text { R), Seec }\end{array}$ & ODD \\
\hline
\end{tabular}


Table 6. Eigenvalue, percentage variation, cumulative variance and correlation between the original variables and four factors derived from principal component $(\mathrm{PC})$ analysis

\begin{tabular}{|c|c|c|c|c|}
\hline Variable & PC1 & PC2 & PC3 & PC4 \\
\hline Shoot length & 0.742 & -0.646 & 0.171 & -0.043 \\
\hline Root length & 0.728 & -0.159 & 0.648 & 0.074 \\
\hline Shoot fresh weight & 0.972 & -0.225 & -0.049 & 0.031 \\
\hline Root fresh weight & 0.810 & 0.341 & 0.447 & 0.116 \\
\hline Shoot dry weight & 0.554 & -0.219 & -0.709 & 0.376 \\
\hline Root dry weight & 0.713 & 0.346 & 0.548 & 0.188 \\
\hline Seedling fresh weight & 0.987 & -0.120 & 0.030 & 0.048 \\
\hline Seedling dry weight & 0.720 & -0.424 & -0.484 & 0.245 \\
\hline Germination percent & 0.603 & 0.633 & 0.123 & -0.322 \\
\hline Germination rate & 0.795 & 0.529 & -0.146 & 0.167 \\
\hline Mean germination time & -0.871 & -0.365 & 0.252 & -0.049 \\
\hline Seed vigour indicator & 0.855 & -0.177 & 0.483 & -0.070 \\
\hline Salt tolerance index & 0.653 & 0.741 & -0.149 & 0.043 \\
\hline Hydrogen peroxide & -0.380 & 0.885 & 0.263 & -0.051 \\
\hline Lipid peroxidation & -0.517 & 0.679 & 0.216 & 0.475 \\
\hline Total soluble proteins & 0.554 & -0.619 & -0.081 & -0.549 \\
\hline Guaiacol peroxidase & 0.218 & 0.912 & -0.071 & -0.341 \\
\hline Ascorbate peroxidase & 0.391 & 0.303 & -0.837 & -0.095 \\
\hline Superoxide dismutase & 0.119 & 0.816 & -0.393 & -0.178 \\
\hline Eigenvalue & 8.84 & 5.58 & 3.03 & 1.09 \\
\hline$\%$ of variance & 46.51 & 29.37 & 15.93 & 5.72 \\
\hline Cumulative variance $\%$ & 46.51 & 75.88 & 91.81 & 97.53 \\
\hline
\end{tabular}

PC1, the first principle component; PC2, the second principal component; PC3, the third principal component and PC4, the fourth principle component.

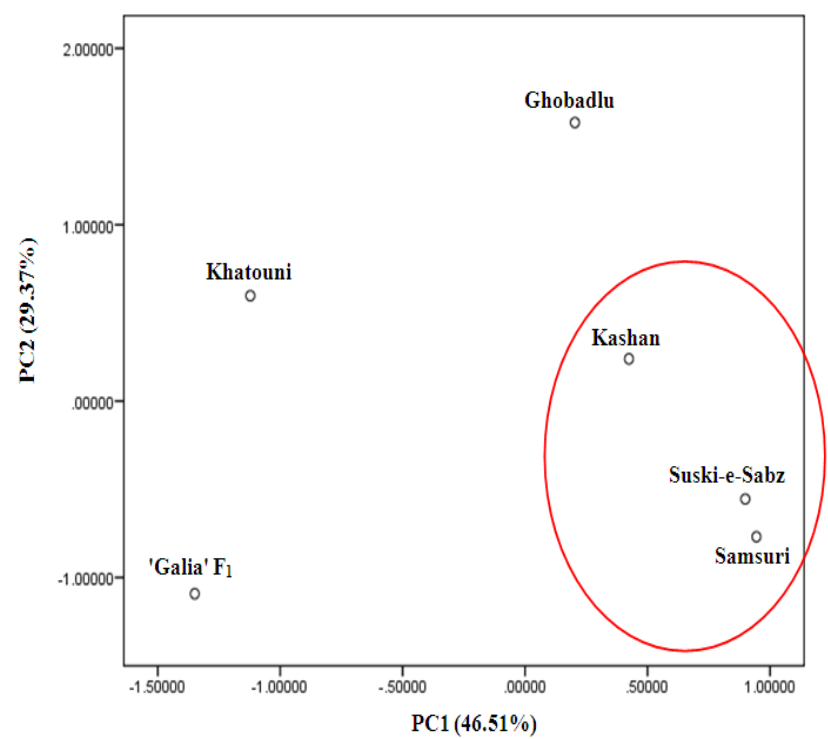

Fig. 4. The score plot for the first two principal components (jointly explaining $75.88 \%$ of the variance) of all melon landraces. The red oval line indicating that the responses of Kashan, Suski-e-Sabz and Samsuri landraces to salinity treatments were similar to somewhat.

landraces, the 'Galia' F1 had the lowest seedling fresh weight $(0.18 \mathrm{~g})$, seed vigour indicator $(8790.44)$, germination rate (2.69 seeds day $\left.{ }^{-1}\right)$, POD activity $\left(84.94 \mu \mathrm{M} \mathrm{min}^{-1} \mathrm{mg}^{-1}\right.$ protein) and the highest mean germination time ( 4.67 day). The highest shoot fresh weight $(0.28 \mathrm{~g})$ and seedling fresh weight $(0.36 \mathrm{~g})$ and the lowest mean germination time (2.38 day) were in Kashan and Suski-e-Sabz landraces, respectively. The Samsuri had the highest seed vigour indicator (14826.66) and the lowest SOD activity (1.01 $\mathrm{U} \mathrm{mg}^{-1}$ protein), in comparing with other landraces.

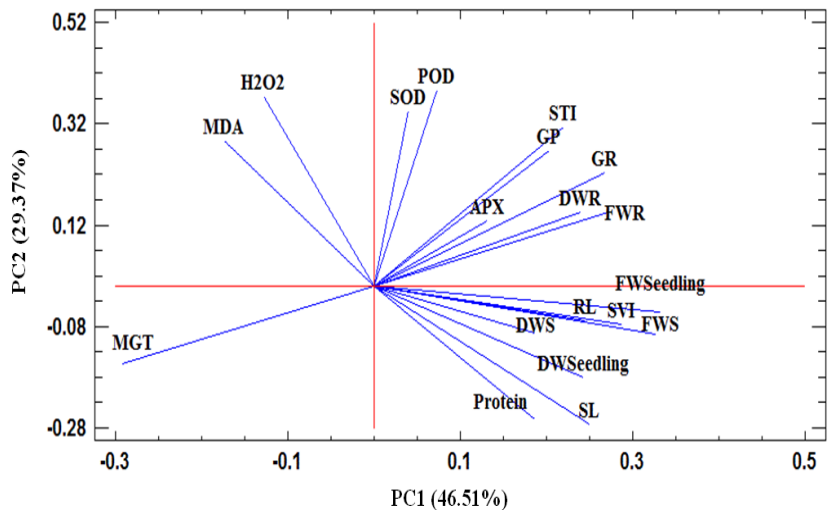

Fig 5. The loading plot of all the measured variables included in the principal components analysis. Considered variables: shoot length (SL), root length (RL), fresh weight of shoot (FWS), fresh weight of root (FWR), dry weight of shoot (DWS), dry weight of root (DWR), fresh weight of seedling (FWSeedling), dry weight of seedling (DWSeedling), germination percent (GP), germination rate (GR), mean germination time (MGT), seed vigour indicator (SVI), salt tolerance index (STI), hydrogen peroxide $\left(\mathrm{H}_{2} \mathrm{O}_{2}\right)$, lipid peroxidation (MDA), total soluble proteins (protein), guaiacol peroxidase (POD), ascorbate peroxidase (APX) and superoxide dismutase (SOD)

One interesting interpretation of the PCA score plot (Fig. 4) is that the Kashan, Suski-e-Sabz and Samsuri landraces were placed very closely indicating that the responses of these landraces to salinity treatments were similar to somewhat. With regard to this fact that the origin of these landraces is in the centre of Iran and at the margin of Dasht-e Kavir desert (Table 1), it can be concluded that the same climate and environmental conditions in this geographical area had similar effects on their reactions to salinity, as an adaptation strategy over the years. 
The loading plot of all the measured variables included in the principal components analysis was shown in the Fig. 5. Variables with higher scores on $\mathrm{PCl}$ are the shoot and root length, shoot and root fresh weight, shoot and root dry weight, seedling fresh and dry weight, germination percent, germination rate, mean germination time, seed vigour indicator, salt tolerance index, total soluble proteins and lipid peroxidation. On PC2, the highest scores were due to the shoot length, germination percent, germination rate, salt tolerance index, hydrogen peroxide content, lipid peroxidation, total soluble proteins, guaiacol peroxidase and superoxide dismutase activities. It is worthwhile that the PC3 exhibited the largest loading value in ascorbate peroxidase activity (Table 6).

\section{Discussion}

Salinity involves osmotic and ionic stresses and the suppression of growth is directly contingent on the total soluble salt concentration and the high osmotic pressure of the solutions. The detrimental effect can be seen at the whole-plant level as plant death or decreased growth and productivity (Kusvuran, 2012).

According to the above results, it is clear that all the studied traits were significantly affected by the increase of salt concentrations. Considering all landraces across all treatments, increased $\mathrm{NaCl}$ significantly had a negative effect on the shoot and root length, shoot and root fresh weight, shoot and root dry weight, seedling fresh and dry weight, germination percent, germination rate, mean germination time, seed vigour indicator and salt tolerance index.

Across all landraces, salt tolerance index decreased as the salt concentrations increased and it was $91.15 \%, 88.07 \%$, $80.16 \%$ and $78.99 \%$ at $20,40,60$ and $80 \mathrm{mM} \mathrm{NaCl}$, respectively. The Ghobadlu and 'Galia' F1 Hybrid, showed the highest and lowest salt tolerance index, $97.57 \%$ and $68.64 \%$, respectively. Kokten et al. (2010) determined that tolerant landraces showed higher salt tolerance indices than sensitive ones. Also, Kusvuran et al. (2007b) reported that the plant stress index value was as an effective parameter for the determination of salt tolerance in melons. Zabihi-eMahmoodabad et al. (2011) reported a decrease with increasing salinity in the shoot and root fresh and dry weights and other studies also reported this trait as the main indicator of salinity tolerance. Moreover, Carpici et al. (2009) detected a negative relationship between the vegetative growth parameters and increasing salinity. They also reported that the effects of different salt concentrations on the salt tolerance indices of cultivars were of importance.

In our experiment, lipid peroxidation measured as malondialdehyde (MDA) production during the oxidation of polyunsaturated fatty acids, which is considered to be an indicator of oxidative damage from stress. The data showed that MDA content was remarkably increased by salinity increase in all landraces.

Among the active oxygen species, superoxide anions $\left(\mathrm{O}_{2}{ }^{-}\right)$are generated in biotic and abiotic stresses, such as salt stress. SODs are metalloproteins that catalyze the dismutation reaction of two superoxide free radicals to one molecule of $\mathrm{O}_{2}$ and one molecule of $\mathrm{H}_{2} \mathrm{O}_{2}$ (Elavarthi and Martin, 2010). In our study, increasing $\mathrm{NaCl}$ concentration led to a significant increase in SOD activity of melon seedlings, that the highest activity was found in the

\section{Ghobadlu landrace.}

$\mathrm{H}_{2} \mathrm{O}_{2}$ is a product of many cellular reactions, e.g., photorespiration and respiration, as well as the activity of SOD. It is established that $\mathrm{H}_{2} \mathrm{O}_{2}$, a representative ROS, accumulates more in germinating seeds under saline conditions than in nonsaline conditions (Lee et al., 2010). In this study the content of $\mathrm{H}_{2} \mathrm{O}_{2}$ significantly increased as the salinity level increased (Table 3).

Detoxification of $\mathrm{H}_{2} \mathrm{O}_{2}$ is enhanced under the same stressful conditions that enhance the production of ROS. We studied two enzymes (POD and APX) that convert $\mathrm{H}_{2} \mathrm{O}_{2}$ to $\mathrm{H}_{2} \mathrm{O}$. Our results showed that, these enzyme activities in all landraces across to all treatments were increased by salinity. These enzymes were previously reported to be important in salt tolerance in melon (Yasar et al., 2006; Kusvuran et al., 2007a). Protecting against salt-stress damage could be a result of higher levels of POD and APX enzymes.

The increase in POD activity was most dramatic in the Ghobadlu landrace, so that it was 1.93, 2.1, 2.41, 2.65 and 3.94 fold more than Khatouni, Kashan, Suski-e-Sabz, Samsuri and 'Galia' F1 landraces, respectively (Fig. 3A).

As regards the highest POD, SOD activities and salt tolerance index (97.57\%) were observed in the Ghobadlu landrace, it can be recommended as a local most promising variety, seeds of which can germinate in the presence of $80 \mathrm{mM}$ $\mathrm{NaCl}$. This result is accommodated with the PCA score plot (Fig. 4) that the Ghobadlu landrace was placed separately in the positive area of the first principal component (PC1) and second principal component (PC2). It is worthwhile that this landrace is cultivated in the northwestern of Iran and eastern margin of Lake Urmia, the largest lake in the Middle East and the sixth largest saltwater lake on earth.

In this study, the performance of 'Galia' F1 hybrid, as a salttolerant cultivar (Yasar et al., 2006), was not remarkable under salinity stress. It had the lowest fresh weight seedling $(0.18 \mathrm{~g})$, germination percent $(83.4 \%)$, germination rate $\left(2.69\right.$ seeds day $\left.^{-1}\right)$, seed vigour indicator $(8790.44)$, POD activity $\left(84.94 \mu \mathrm{M} \mathrm{min}^{-1}\right.$ $\mathrm{mg}^{-1}$ protein) and the highest mean germination time ( 4.67 day), in comparing with Iranian melon landraces. This is in contrast with the results of Yasar et al. (2006), which reported that 'Galia' F1 is a salt-tolerant cultivar, because it exhibited higher enzymatic and non-enzymatic anti-oxidant defence systems in response to salt induced oxidative stress in the 5 weeks seedlings. These inconsistent results can be explained by the Del Amor et al. (1999) report, that the response of melons to salinity depends on the duration of exposure to saline water and the inhibitory effects of salinity will be progressively lessened when salinity is imposed at later growth stages.

\section{Conclusions}

The different responses to salinity stress between the landraces could be used to assess salt tolerance potentialities and our study suggests that the salt tolerance index (STI) and principal component analysis (PCA) could be used as accurate and easy indicators when screening melons for salt tolerance. New tests will be needed to validate these indicators for higher salinity levels and also at the later growth stages. Also, the identified landraces with superior salinity tolerance in the germination stage will be crossed with existing melon cultivars to improve salinity tolerance. 


\section{References}

Abdul-Baki AA, Anderson JD (1973). Vigor determination in soybean seed by multiple criteria. CropScience 13(6):630-633

Alexieva V,Sergiev I, MapelliS, KaranovE(2001). The effect of drought and ultraviolet radiation on growth and stress markers in pea and wheat. Plant, Cell \&Environment 24(12):1337-1344.

Allen RD (1995). Dissection of oxidative stress tolerance using transgenic plants. Plant Physiology 107(4):1049-1054.

Bajji M, Kinet JM, Lutts S (2002). Osmotic and ionic effects of $\mathrm{NaCl}$ on germination, early seedling growth and ion content of Atriplex halimus (Chenopodiaceae). Canadian Journal ofBotany 80(3):297-304.

Bradford MM (1976). A rapid and sensitive method for the quantitation of microgram quantities of protein utilizing the principle of protein-dye binding. Analytical Biochemistry 72(1-2):248-254.

Cantliffe DJ (1991). Benzyladenine in priming solution reduces thermodormancy oflettuce seeds. HortTechnology 1(1):95-97.

Carpici EB, Celik N, Bayram G (2009). Effects of salt stress on germination of some maize (Zea mays L.) cultivars. African Journal of Biotechnology 8(19):4918-4922.

Cuartero J, Bolarin MC, Asins MJ, Moreno V (2006). Increasing salt tolerance in the tomato. Journal of Experimental Botany 57(5):10451058.

Del Amor FM, Martinez V, Cerda A (1999). Salinity duration and concentration affect fruit yield and quality, and growth and mineral composition of melon plants grown in perlite. HortScience 34(7):1234 1237.

Dhindsa RS, Plump-Dhindsa P, Thorpe TA (1981). Leaf senescence: Correlated with increased levels of membrane permeability and lipid peroxidation, and decreased levels of superoxide dismutase and catalase. Journal of Experimental Botany 32(1):93-101.

Elavarthi S, Martin B (2010). Spectrophotometric assays for antioxidant enzymes in plants. In: Sunkar R (Ed). Plant Stress Tolerance: Methods and Protocols. Springer, New York pp 273-280.

El Goumi Y, Fakiri M, Lamsaouri O, Benchekroun M (2014). Salt stress effect on seed germination and some physiological traits in three Moroccan barley (Hordeum vulgare L.) cultivars. Journal of Materials and Environmental Science 5(2):625-632.

FAO(2010).FAOSTAT agricultural database http://apps.fao.org.

Franco JA, Fernandez JA, Banon S (1997). Relationship between the effects of salinity on seedling leaf area and fruit yield of six muskmelon cultivars. HortScience 32(4):642-644.

Heath RL, Packer L (1968). Photoperoxidation in isolated chloroplasts: I. Kinetics and stiochiometry of fatty acid peroxidation. Archives of Biochemistry and Biophysics 125(1):189-198.

Hemeda HM, Kelin BP (1990). Effects of naturally occurring antioxidants on peroxidase activity of vegetable extracts. Journal of Food Science 55(1):184-185.

Khanna-Chopra R, Selote DS (2007). Acclimation to drought stress generates oxidative stress tolerance in drought-resistant than susceptible wheat cultivar under field conditions. Environmental and Experimental Botany 60(2):276-283.
Kokten K, Karakoy T, Bakoglu A, Akcura M (2010). Determination of salinity tolerance of some lentil (Lens culinaris M.) varieties. Journal of Food, Agriculture \& Environment 8(1):140-143.

Korkmaz A (2005). Inclusion of acetyl salicylic acid and methyl jasmonate into the priming solution improves low temperature germination and emergence of sweet pepper seeds. HortScience 40(1):197-200.

Kusvuran S (2012). Ion regulation in different organs of melon (Cucumis melo L.) genotypes under salt stress. International Journal of Agriculture \& Biology 14(1):141-144.

Kusvuran S, Ellialtioglu S, Yaser F, Abak K (2007a). Effects of salt stress on ion accumulation and activity of some antioxidant enzymes in melon (Cucumis melo L.). Journal of Food Agriculture and Environment 5(2):351-354.

Kusvuran S, Yasar F, Ellialtioglu S, Abak K (2007b). Utilizing some of screening methods in order to determine of tolerance of salt stress in the melon (Cucumis melo L.). Research Journal of Agriculture and Biological Sciences 3(1):40-45.

Lee S, Kim SG, Park CM (2010). Salicylic acid promotes seed germination under high salinity by modulating antioxidant activity in Arabidopsis. New Phytologist 188(2):626-637.

Loggini B, Scartazza A, Brugnoli E, Navari-Izzo F (1999). Antioxidative defense system, pigment composition and photosynthetic efficiency in two wheat cultivars subjected to drought. Plant Physiology 119(3):1091-1100.

Mangal JL, Hooda PS, Lal S (1988). Salt tolerance of five muskmelon cultivars. The Journal of Agricultural Science 110(3):641-643.

Meiri A, Hoffman G, Shannon MN, Poss J (1982). Salt tolerance of two muskmelon cultivars under two solar radiation levels. Journal of the American Society for Horticultural Science 107:1168-1172.

Mendlinger S, Pasternak D (1992). Screening for salt tolerance in melons. HortScience 27(8):905-907.

Munns R(2002). Comparative physiology of salt and water stress. Plant, Cell \&Environment 25(2):239-250.

Munns R, Tester M (2008). Mechanisms of salinity tolerance. Annual Review of Plant Biology 59:651-681.

Nakano Y, Asada K (1981). Hydrogen peroxide is scavenged by ascorbatespecific peroxidase in spinach chloroplasts. Plant \& Cell Physiology 22(5):867-880.

Pitrat M, Hanelt P, Hammer K (2000). Some comments on infraspecific classification of cultivars of melon. Acta Horticulturae 510:29-36.

Yasar F, Kusvuran S, Ellialtioglu S (2006). Determination of anti-oxidant activities in some melon (Cucumis melo $\mathrm{L}$.) varieties and cultivars under salt stress. Journal of Horticultural Science and Biotechnology 81(4):627-630.

Zabihi-e-Mahmoodabad R, Jamaati-e-Somarin S, Khayatnezhad M, Gholamin $R$ (2011). The study of effect salinity stress on germination and seedling growth in five different genotypes of wheat. Advances in Environmental Biology 5(1):177-179. 